\title{
PERCEPÇÃO DE EMPREGABILIDADE DE TRABALHADORES EM CONDIÇÕES INCERTAS DO MERCADO DE TRABALHO
}

\section{PERCEPTION OF EMPLOYABILITY OF WORKERS IN UNCERTAIN LABOR MARKET CONDITIONS}

\section{GRASIELA TORRICO}

Pesquisadora do Laboratório de Informação e Orientação Profissional (LIOP) - UFSC Mestre em Psicologia - Universidade Federal de Santa Catarina (UFSC)

Orcid: https://orcid.org/0000-0002-1200-9876

E-mail: grasit@hotmail.com

Centro de Filosofia e Ciências Humanas - UFSC

Campus Universitário Reitor João David Ferreira Lima, s/n, CEP: 88040-900, Trindade - Florianópolis/SC

\section{MAIANA FARIAS OLIVEIRA NUNES}

Docente do Departamento de Psicologia - Universidade Federal de Santa Catarina (UFSC) Doutora em psicologia - Universidade São Francisco

Orcid: https://orcid.org/0000-0003-4891-5982

E-mail:maiananunes@mac.com

\section{ROBERTO MORAES CRUZ}

Docente do Departamento de Psicologia - Universidade Federal de Santa Catarina (UFSC) Doutor em Engenharia de Produção - Universidade Federal de Santa Catarina (UFSC)

Orcid: https://orcid.org/0000-0003-4671-3498

E-mail: robertocruzdr@gmail.com

Submissão: 18/08/2021. Revisão: 05/09/2021. Aceite: 29/10/2021. Publicação: 01/02/2022. DOI: http://dx.doi.org/10.22277/rgo.v15i1.6616

\section{RESUMO}

Objetivo: O objetivo deste artigo foi analisar a produção do conhecimento sobre a percepção de empregabilidade em trabalhadores.

Método / abordagem: Foi realizada uma revisão integrativa sobre a percepção de empregabilidade de trabalhadores no mercado de trabalho contemporâneo, abordando as diversas interferências contextuais, sejam elas internas ou externas e, também, a as relações entre a dinâmica do mercado de trabalho e das inovações tecnológicas nas trajetórias de carreira.

Principais resultados: A empregabilidade não se constitui apenas um resultado, mas como a quantidade de recursos de empregabilidade que as pessoas possuem. Percepções mais fortes de empregabilidade resultam em uma perspectiva mais positiva no trabalho, refletindo na avaliação da volatilidade e inseguranças do mercado de trabalho como menos ameaçadoras. Além disso, mostra-se positivamente associada à satisfação com a vida, controle, otimismo,

Este é um artigo publicado em acesso aberto (Open Access) sob a licença Creative Commons Attribution, que permite uso, distribuição e reprodução em qualquer meio, sem restrições desde que o trabalho original seja corretamente citado. 
suporte no desenvolvimento e sucesso na carreira, bem como com engajamento, comprometimento organizacional e níveis mais baixos de exaustão no trabalho.

Contribuições metodológicas / sociais / gerenciais: Foi realizada uma discussão sobre a percepção de empregabilidade em seus fundamentos teóricos e perspectivas analíticas de investigação, considerando a perceptiva individual e a influência de fatores contextuais. A revisão integrativa buscou exaurir os principais aspectos salientes ao entendimento do fenômeno da empregabilidade e sua relação com o repertório socioprofissional dos trabalhadores e os desafios e oportunidades do mundo do trabalho.

Originalidade / relevância: $O$ estudo contribui ao examinar as diferenças entre a empregabilidade e a percepção de empregabilidade para avançar no entendimento de seus antecedentes e repercussões nas trajetórias de carreira.

Palavras-chave: Percepção de empregabilidade. Carreira. Trabalhadores.

\begin{abstract}
Purpose: The aim of this article was to analyze the production of knowledge about the perception of employability among workers.

Method / approach: An integrative review was carried out on the perception of employability of workers in the contemporary labor market, addressing the various contextual interferences, whether internal or external, and the relationships between the dynamics of the labor market and technological innovations in career trajectories.

Main findings: Employability is not just a result but is also considered the amount of employability resources that people have. Stronger perceptions of employability result in a more positive perspective at work, reflecting in the assessment of volatility and insecurities in the labor market as less threatening. In addition, it is positively associated with life satisfaction, control, optimism, support in career development and success, as well as engagement, organizational commitment, and lower levels of exhaustion at work.

Methodological / social / managerial contributions: A discussion was carried out on the perception of employability in its theoretical foundations and analytical research perspectives, considering the individual perception and the influence of contextual factors. The integrative review sought to exhaust the main salient aspects for understanding the phenomenon of employability and its relationship with the socio-professional repertoire of workers and the challenges and opportunities in the world of work.
\end{abstract}

Originality / relevance: The study contributes by examining the differences between employability and the perception of employability to advance the understanding of their antecedents and repercussions on career trajectories.

Keywords: Perceived employability. Career. Workers.

\title{
1 INTRODUÇÃO
}

O conceito de empregabilidade, de uma forma geral, corresponde à capacidade de ser empregável, ou seja, de obter emprego ou trabalho, nele permanecer ou progredir, levandose em conta as exigências de qualificações, habilidades e conhecimentos ofertadas pelo mercado de trabalho (Hillage \& Pollard, 1998; Veld et al., 2015). Nessa direção, a empregabilidade diz respeito a um conjunto de requisitos, atributos e aprendizagens pessoais 
que se estendem ao mundo do trabalho, associando-se às trajetórias ocupacionais e às trajetórias de carreiras (Rothwell \& Arnold, 2007).

A empregabilidade é retratada, historicamente, como uma condição objetiva das pessoas em se adequarem às exigências do mundo do trabalho, com base em suas qualificações, necessidades, interesses, e meta-competências para fatores externos, sustentado em valores pessoais, atribuição de significado do trabalho e de bem-estar dos indivíduos (Di Fabio, 2017). Esse processo é aperfeiçoado conforme o grau de aprendizado contínuo de trabalhadores e de flexibilidade e maturidade das organizações (Savickas et al., 2009).

O fenômeno da empregabilidade é compreendido como uma relação entre quatro categorias de estudo da relação pessoa-trabalho: fatores individuais (atitudes, habilidades, interesses, motivos, expectativas), circunstâncias individuais (oportunidades para o aprendizado e desenvolvimento de habilidades), sistema de apoio facilitador (meio familiar e organizacional) e mercado de trabalho (ofertas de emprego e serviços). Essas categorias são utilizadas, em diferentes pesquisas, para mensurar e produzir indicadores sobre empregabilidade percebida (Jackson \& Wilton, 2017), empregabilidade sustentável (Van der Klink, 2016), habilidades de empregabilidade (Suleman, 2018), expectativas de empregabilidade (Tsitskari et al., 2017), dentre outros.

É importante, contudo, diferenciar empregabilidade e contrato de trabalho. O contrato de trabalho "solidifica" uma relação de trabalho com uma organização, enquanto a empregabilidade é a capacidade do trabalhador em se manter na possibilidade real de ser contratado, tendo em vista suas qualificações e habilidades e às oportunidades de vínculo com o mercado de trabalho (Dries et al., 2014).

Frente ao cenário de incerteza do mundo do trabalho e de demanda de autogestão da carreira, cresce a importância de se estudar as ações empreendidas pelas pessoas para desenvolver habilidades e buscar conhecimentos favoráveis à sua inserção ou manutenção no mercado de trabalho, seja ele formal ou informal (Rueda et al., 2004). Do ponto de vista da gestão de pessoas, a implementação de políticas e práticas direcionadas à empregabilidade é vista como uma estratégia importante para promover o desenvolvimento de transições de carreira, o comprometimento organizacional e a capacidade de facilitar mudanças em diferentes níveis das organizações (Forrier \& Sels, 2003; Ellinger \& Ellinger 2014; Veth et al., 2015; Di Fabio, 2017).

Na perspectiva individual, a empregabilidade envolve um conjunto de convicções pessoais quanto à capacidade pessoal em conquistar, manter ou substituir um emprego, pois, nesse caso, é o próprio indivíduo que identifica o seu grau de empregabilidade (Berntson \& Marklund, 2007; Berntson et al., 2006). Contudo, é importante ressaltar as diversas influências contextuais na empregabilidade percebida pelo trabalhador, uma vez que esses comportamentos relacionados à empregabilidade são influenciados pelo ambiente e o influenciam em uma relação de influência mútua. Adicionalmente, o contexto irá influenciar no sentido de fornecer oportunidades de desenvolvimento pessoal/profissional em níveis e em quantidades variadas (Bandura, 1997).

A percepção de empregabilidade continua sendo um conceito relativamente pouco pesquisado em trabalhadores, na perspectiva de analisar o significado das experiências, aspirações e convicções dos trabalhadores frente à competição no mundo do trabalho (Kinnunen et al., 2011; Rothwell et al., 2009). Refletir sobre o fenômeno da empregabilidade e a da sua percepção pelo trabalhador auxilia na compreensão dos momentos de flutuações 
e incertezas, perspectivas e desafios nas trajetórias profissionais, na medida em que as decisões de carreira não são consideradas um evento único, mas irão se suceder, muitas vezes, ao longo do ciclo de vida das pessoas (Kim et al., 2014).

Com base nessas contribuições, o objetivo deste artigo foi analisar a produção do conhecimento sobre a percepção de empregabilidade em trabalhadores. Nesse âmbito, são destacadas as características conceituais e as perspectivas de abordagens da percepção de empregabilidade de trabalhadores, considerando a influência da dinâmica dos processos produtivos, do mercado de trabalho e das inovações tecnológicas no desenvolvimento de habilidades e das trajetórias de carreira.

\section{ASPECTOS METODOLÓGICOS}

Foi realizado um levantamento de literatura, por meio de uma revisão integrativa, uma forma de pesquisa que proporciona que sejam gerados novos conhecimentos, estruturas e perspectivas sobre um determinado problema de investigação (Hopia et al., 2016). Buscou-se explorar perspectivas e argumentos científicos que permitam esclarecer e aperfeiçoar o entendimento sobre os aspectos que compõe a percepção de empregabilidade.

A revisão integrativa da literatura seguiu as indicações de Torraco (2016). A cobertura da literatura utilizada foi exaustiva, com citação seletiva, sem critérios de limites de temporalidade para capturar a amplitude da literatura relevante, além de observar parâmetros específicos para acessar os estudos independente da sua temporalidade. Os procedimentos metodológicos incluíram inicialmente a construção de um protocolo para selecionar os tópicos que seriam utilizados na busca de dados, bem como os critérios de inclusão/exclusão, bases de dados e criação da estratégia de busca.

As buscas foram realizadas nos meses de julho e agosto de 2020 e foram consideradas tanto pesquisas empíricas quanto teóricas. Considerou-se para critérios de inclusão estudos que abordassem a temática de 'Percepção de Empregabilidade' com o público-alvo de trabalhadores, sendo excluídos estudos que não fossem ao encontro desse propósito.

A estratégia de busca incluiu termos em inglês mais recorrentes que foram levantados após uma busca prévia em artigos e produções científicas da área, tendo sido identificadas as bases de dados que obtiveram um maior volume de publicações com a temática proposta. Assim, a operacionalização da busca englobou todos os periódicos da base de dados nacional SciELO e as bases internacionais Science Direct e Web of Science, por meio dos descritores "employability" AND ("perception" OR "self-perceived" OR "perceived" OR "perceived by the individual"). Foram selecionados os campos de busca: título, resumo e palavras-chave, restringindo o tipo de documento apenas para artigos.

Durante a realização da pesquisa, a tabulação dos dados ocorreu por meio de uma planilha no Microsoft Office Excel para o preenchimento de todos os itens para posterior análise, constando os nomes dos artigos, revistas, ano de publicação, resumo, público-alvo e local da pesquisa. Finalizado este processo, foi possível identificar quais artigos se adequavam ao foco da pesquisa e quais seriam eliminados. Foram selecionados um total de 186 documentos, assim distribuídos: Scielo $(n=31)$, Web of Science $(n=135)$ e Science Direct $(n=$ 20), conforme mostra a Figura 1.

Para a análise dos documentos selecionados foi primeiramente realizada uma filtragem de documentos duplicados entre as bases (16), restando 170. Em seguida, com base na leitura dos resumos/abstracts, foram excluídos 23 documentos que não adotavam o 
formato de artigos ou não contemplavam o tema, em termos de objetivo e método. Em seguida, em novo filtro de decisão, foram excluídos 84 artigos que direcionavam seus estudos para outros aspectos não relacionados com o propósito desta pesquisa (24), ou com enfoque na empregabilidade objetiva, tendo como público-alvo estudantes (10) ou trabalhadores (13), ou ainda com o enfoque na temática de percepção de empregabilidade, mas com outros públicos pesquisados como estudantes (53), detentos (4), desempregados (2) ou pacientes médicos (1). Com isso, foram eleitas 63 produções científicas para a revisão integrativa, contemplando os anos de 2006 (1), 2007 (3), 2009 (1), 2010 (2), 2011 (4), 2012 (3), 2013 (3), 2014 (6), 2015 (9), 2016 (4), 2017 (6), 2018 (7), 2019 (9) e 2020 (5).

Figura 1

Fluxograma das decisões da revisão integrativa

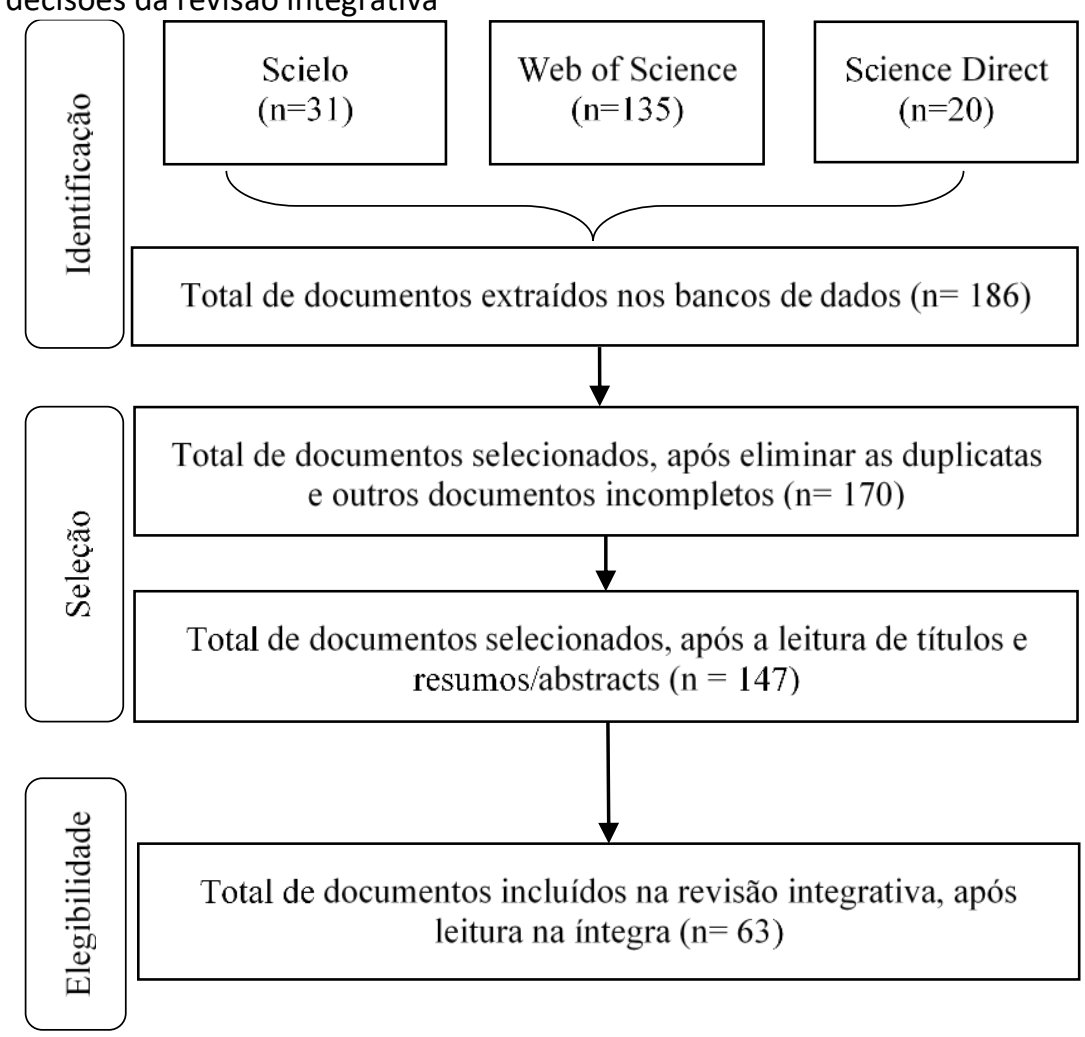

Dos artigos eleitos, 12 deles tiveram origem na Bélgica, 6 na Itália, 6 na Finlândia e 5 na Espanha. Os demais estudos foram identificados em 18 outros países, embora nenhum deles com origem no Brasil. Predominaram os estudos eram transversais, sendo apenas 6 longitudinais, e somente 2 artigos apresentaram uma abordagem qualitativa. Os estudos buscaram discutir o conceito de percepção de empregabilidade em conjunto com os construtos: autoeficácia, insegurança no trabalho, satisfação com o trabalho, bem-estar, aprendizagem e saúde mental. Com base na análise desses estudos, foram destacados eixos de discussão para a presente revisão, salientando as principais contribuições à investigação da percepção de empregabilidade.

\section{RESULTADOS E DISCUSSÃO}

Será realizada, inicialmente, uma descrição e localização temporal do contexto de trabalho e um breve apontamento sobre carreira. Logo após as definições de percepção de empregabilidade e seus fundamentos teóricos serão apresentados e, por fim, a seção final 
Percepção de empregabilidade de trabalhadores em condições incertas do mercado de trabalho

ilustrará a relevância, sugestões de intervenções e de futuros estudos a respeito da percepção de empregabilidade com o público de trabalhadores.

\subsection{CONTEXTO DO TRABALHO CONTEMPORÂNEO E CARREIRA}

O cenário do mundo do trabalho mudou de um lugar em que o crescimento econômico anual produzia novos empregos de modo suficiente, para um mundo econômico desafiador em que os poucos empregos não suprem mais a grande demanda vigente. A predominância de aparatos tecnológicos que auxiliam a agilizar a produção e otimizar os resultados de trabalho, assim como locais de trabalho que influenciam para a aceleração de mudanças, alteram significativamente o ambiente no qual os trabalhadores desenvolvem suas carreiras e, principalmente, elaboram suas decisões (Cruz et al., 2020).

O contexto dinâmico do trabalho, assim como a crescente complexidade e imprevisibilidade das carreiras, associa-se à influência dos fatores ambientais, culturais, tecnológicos e socioeconômicos no desenvolvimento das aprendizagens e trajetórias profissionais, impactando na percepção de empregabilidade (Mourão \& Fernandes, 2020). Portanto, é cada vez mais provável que a percepção da empregabilidade seja susceptível a eventos aparentemente imprevisíveis, situados no contexto do trabalho e dos processos produtivos (Akkermans et al., 2018), mas que promovem um impacto significativo nas carreiras das pessoas.

Algumas condições contextuais tendem a desestabilizar o mercado de trabalho como, por exemplo, recessões econômicas ou crises no mercado financeiro. De igual modo, a demanda pela criação de novas tecnologias, de empreendimentos sustentáveis ambientalmente, assim como a transição demográfica de envelhecimento da população ou de expansão da base da pirâmide etária, representa uma agenda de desafios à promoção de soluções compatíveis às necessidades do mercado de trabalho e que garantam a proteção da saúde e a integridade dos trabalhadores (Maranhão \& Savino, 2019). Dessa forma, além da responsabilidade do indivíduo pelo desenvolvimento da sua trajetória de carreira, é relevante apontar a necessidade de apoio de políticas públicas de incentivos à qualificação profissional e à melhoria das condições de trabalho, que possam auxiliar os trabalhadores na inserção ou reinserção no mercado de trabalho (Façanha Neto \& Façanha, 2020).

Em termos de desenvolvimento de carreira, tem-se acompanhado o crescimento de trajetórias descontínuas, praticadas de maneira informal e sem emprego remunerado como base (Antunes, 2015). Nos tempos atuais, a gestão da carreira foi transferida das organizações para o trabalhador, ou seja, cada vez mais o indivíduo é considerado o responsável pelo desenvolvimento de sua carreira, como também é submetido a administração das influências de diversos contextos em sua vida (Savickas \& Baker, 2005).

A alteração de carreiras organizacionais para carreiras autogerenciadas se mostrou uma crescente necessidade de trabalhadores recém-formados em internalizar a escassez de carreiras tradicionais e desenvolver a mentalidade e as habilidades para identificar e navegar efetivamente em carreiras mais adequadas às circunstâncias, objetivos e capacidades pessoais (Jackson \& Tomlinson, 2020). Dessa maneira, mesmo no contexto de mercado incertos, é importante que os profissionais avaliem suas habilidades e perspectivas de manutenção do emprego ou inserção em novas oportunidades de trabalho e renda, diante das opções disponíveis ou a serem geradas no mercado de trabalho. 
Adicionalmente, o surgimento de trabalhos alternativos, estimulados pelas novas tecnologias de comunicação e informação, mediados pela internet, estimulou mudanças na organização do trabalho e na percepção de carreiras. Lent (2018), nesse contexto, destaca três modalidades de trabalhos alternativos: i) "gig economy": a combinação de empresas com trabalhadores dispostos a se envolver em trabalho temporário, contratual ou freelancer; ii) "peer economy": empresas online que permitem que os trabalhadores forneçam serviços diretamente aos consumidores e iii) "crowdsourcing": um método online para distribuir projetos complexos em grande número de trabalhadores independentes. Essas novas plataformas de trabalho são frequentemente elogiadas por sua flexibilidade, pois permitem que os trabalhadores definam suas próprias horas ou condições de trabalho, contudo geram desafios, tais como a remuneração, geralmente modesta, e a restrição de benefícios (seguro saúde, vale alimentação, dentre outros) ao trabalhador ou ao distribuidor do trabalho (Lent, 2018).

A noção de empregabilidade é frequentemente discutida em situações em que há menor oferta de empregos e quando se analisa a atratividade das ocupações frente à dinâmica do mercado de trabalho (Rothwell \& Arnold, 2007; Fugate et al., 2004). A maneira como o trabalhador avalia o mercado de trabalho, também, determina como ele classifica sua empregabilidade percebida e, em um ambiente de mercado de trabalho incerto, a empregabilidade percebida tem grande importância para promover estratégias de enfrentamento (Berntson et al., 2006).

A avaliação do mercado de trabalho tem uma associação significativa com a autopercepção da empregabilidade, existindo um consenso na literatura de que fatores ambientais e pessoais devem ser integrados ao avançar na compreensão de modelos explicativos da percepção de empregabilidade (Berntson et al., 2006; Qenani et al., 2014). Surpreendentemente, poucas pesquisas explicam sua base ou discutem seu papel em influenciar uma série de comportamentos organizacionais (Fugate et al., 2004). O construto percepção de empregabilidade será alvo do próximo tópico.

\subsection{PERCEPÇÃO DE EMPREGABILIDADE}

O termo empregabilidade possui mais de uma conceituação, e passou a ser utilizado com maior frequência como uma alternativa de compreensão conceitual sobre o enfrentamento dos trabalhadores às contínuas transformações impostas pelo mercado de trabalho. O conceito inicial de empregabilidade entrou em uso por volta de 1955, e a ênfase incidia sobre a aquisição de uma ocupação estável (Forrier \& Sels, 2003).

Na década de 1970, o foco dado ao conceito residia na aquisição de habilidades técnicas que permitissem os trabalhadores adquirirem ou manterem um emprego (Peixoto et al., 2015). A partir da década de 1980, o movimento foi em direção à configuração de múltiplas formas de inserção e de vinculação do trabalhador à organização (Berntson et al., 2006). Em meados da 1990 em diante a empregabilidade passou a ser destacada não somente como um fenômeno socioeconômico, mas também associado ao desenvolvimento da carreira dos trabalhadores e às mudanças nos arranjos do trabalho interno e externo às organizações (Thijssen et al., 2008).

Uma das definições mais citadas sobre empregabilidade, e em uma perspectiva mais abrangente, pertence a Hillage e Pollard (1998), que entendem a empregabilidade como a capacidade de mobilizar a autossuficiência no mercado de trabalho para que o indivíduo possa realizar seu potencial por meio de um emprego sustentável, ou seja, a ideia dos indivíduos 
ganhando controle de sua carreira (Rothwell, 2015). Contudo, a empregabilidade também depende do conhecimento, habilidades e atitudes do indivíduo, da maneira como usa esses ativos e os apresenta a possíveis empregadores, além de fatores do contexto no qual eles procuram trabalho. Nessa direção, embora de forma sintética, Rothwell e Arnold (2007) sugerem que a empregabilidade possa ser definida como a capacidade de manter o emprego que se tem ou de conseguir o emprego que se deseja. Argumenta-se que quanto maior for a empregabilidade percebida, menor é o sentimento de insegurança e maior a confiança do indivíduo em si próprio, pois consegue desenvolver as suas funções de forma mais competente, ter um desempenho mais elevado e uma maior probabilidade de ascender na sua carreira profissional (Hartley et al., 1990).

Considera-se que a empregabilidade é um fenômeno complexo e multidimensional (Van der Heijde, 2014), ligado ao comportamento do trabalhador e às condições do mercado de trabalho. Nesse caso, pode significar muitas coisas para muitas pessoas, mas todas têm o 'emprego' como um resultado para o conceito (Rothwell, 2015). O conceito de empregabilidade pode incluir dimensões externas ou internas, utilizando indicadores objetivos ou subjetivos, dependendo do propósito a que se propõe estudar e da perspectiva que se quer adotar. Na dimensão externa envolve contextos de aprendizagem ou de trabalho e nas dimensões internas, envolve o reconhecimento de atributos individuais relevantes na procura de emprego e no desempenho profissional (Rothwell et al., 2009). Em síntese, destaca-se que a percepção de empregabilidade é uma condição interpretada pelo trabalhador, no sentido do desenvolvimento e do sucesso na carreira, com base na sua experiência de trabalho, qualificações, competência pessoal, autoconfiança e rede de contatos (Makikangas et al., 2013).

Quanto à exploração da aplicação conceito de empregabilidade, Rothwell (2015) ressalta que é possível aplicar o construto em quatro contextos amplos: i) empregabilidade em políticas públicas (Employability in Public Policy): que normalmente se relaciona com o objetivo de reduzir o desemprego e superar as desvantagens sociais; ii) empregabilidade na educação (Employability in Education): se concentra nos graduados com diploma de bacharel e em como eles se saíram em relação à rápida expansão do setor universitário e às preocupações contínuas sobre a adequação dos graduados ao mundo do trabalho em um contexto frequentemente recessivo; iii) empregabilidade na gestão de recursos humanos (Employability in HR Management): relacionada com estratégias proativas do empregador para contratar funcionários com várias competências e iv) empregabilidade percebida pelo indivíduo (Employability as perceived by the individual): refere-se às autopercepções de empregabilidade dos indivíduos e como eles percebem suas perspectivas atuais e futuras em relação ao emprego.

$\mathrm{Na}$ atualidade, a percepção de empregabilidade obteve uma posição de destaque enquanto elemento decisivo para a proteção do indivíduo no seu relacionamento com o mercado de trabalho. Dado o caráter sem fronteiras das carreiras atuais, no sentido em que muitas barreiras são ultrapassadas dentro e fora da organização, quando as carreiras são menos previsíveis, o conjunto de competências e a empregabilidade percebida torna-se muito importante como ponto de partida para as atividades de carreira e para as futuras oportunidades de emprego (Thijssen et al., 2008).

Os profissionais precisam desenvolver a sua carreira no contexto da variabilidade do mercado de trabalho (Clarke, 2009). Em relação a esse fator contextual, a disponibilidade de empregos ou a relação entre oferta e a exigência afetam a percepção do indivíduo sobre as 
oportunidades de emprego (Forrier et al., 2009). O trabalhador, ao ter ciência dos requisitos do mercado de trabalho, bem como ao se comparar com os demais trabalhadores por meio das suas características, capacidades, conhecimentos, capital social, cria uma referência da sua própria empregabilidade e valor no mercado (Hillage \& Pollard, 1998). Quando os trabalhadores refletem sobre sua empregabilidade e consideram relevantes informações a respeito dos impactos da situação econômica, estas análises sobre períodos econômicos reforçam a conclusão de que educação e região são fatores importantes para a compreensão da empregabilidade percebida dos indivíduos (Berntson et al., 2006). De acordo com Wittekind et al. (2010), trabalhadores que detenham um maior nível de capital cultural e de escolaridade tendem a apresentar escores mais elevados de percepção de empregabilidade. A educação como suporte para a carreira, para o desenvolvimento de capacidades, para o nível de conhecimentos relacionados com o trabalho e a capacidade de encontrar um novo trabalho têm uma relação mais forte e predizem a empregabilidade percebida (Wittekind et al., 2010).

Os empregadores possuem uma posição de destaque para definir ou requerer as habilidades essenciais para o desenvolvimento da sua empresa, assim como prever futuras necessidades de aperfeiçoamento pessoal ou gerencial para lidar com a volatilidade e os desafios impostos pelo mercado de trabalho ao modelo de negócio. Nessa direção, trabalhadores que obtêm e percebem suporte organizacional no desenvolvimento de suas competências e de sua carreira demonstram uma percepção positiva de empregabilidade em comparação com aqueles que não referem suporte do ambiente de trabalho (Drange et al., 2018).

As incertezas do mercado podem ser vistas tanto de forma positiva como negativa. Do ponto de vista negativo, as mudanças de tarefa ou trabalho levam ao aumento do volume de trabalho, de taxas de desemprego e a precarização do trabalho. Pelo lado positivo, trazem resultados como o enriquecimento de trabalho, experiências de novas atividades e oportunidades de desenvolvimento que melhoram a percepção da empregabilidade (Van Dam, 2004). Não é necessariamente a mudança nas atividades de trabalho ou dos fatores contextuais que produzem uma percepção da empregabilidade mais positiva ou negativa, mas sim, a interpretação que os trabalhadores fazem dessas mudanças, no sentido de promover mudanças comportamentais que favorecem as suas trajetórias de carreira e realização profissional.

Complementarmente, a resiliência às instabilidades do mundo do trabalho e aos desafios à carreira fazem parte do processo de construção de repertórios socioprofissionais que refletem na percepção de empregabilidade. Trabalhadores que demonstram otimismo na carreira percebem mais oportunidades no local de trabalho, referem expectativas positivas sobre eventos futuros, concebem mudanças de carreira como desafios e oportunidades de aprender, demonstram confiança em sua capacidade de lidar com estímulos objetivos e afetivos, não tendem a personalizar os motivos de falhas ou erros na carreira, bem como persistem na busca por resultados e objetivos desejados (Carver \& Scheier, 1994).

Um aspecto relevante a ser discutido é se a percepção de empregabilidade possui uma variação ou um grau de importância maior entre trabalhadores temporários ou efetivos, já que há uma maior insegurança ou instabilidade no trabalho temporário. De acordo com De Cuyper et al. (2010), a percepção de empregabilidade é importante para todos os trabalhadores, independentemente do tipo de contrato ou preferências de trabalho, sejam 
Percepção de empregabilidade de trabalhadores em condições incertas do mercado de trabalho

eles temporários ou efetivos, principalmente no mercado de trabalho contemporâneo, onde a insegurança no emprego é uma ameaça universal.

A empregabilidade percebida promove a sensação de estar no controle da carreira, pois estimula a confiança de que uma pessoa pode conseguir um emprego no mercado de trabalho (Moyo \& Theron, 2011). Trabalhadores com mais recursos de empregabilidade percebidos são mais propensos a se verem como atores principais, responsáveis na exploração e criação de oportunidades de carreira, dependendo menos da organização para estabelecer uma estrutura para seus objetivos de carreira a longo prazo (Baruch, 2001; De Vos \& Soens, 2008).

Nessa direção, nutrir a empregabilidade percebida repercute no aumento da autoconfiança dos trabalhadores, para se manter ativo no mercado de trabalho. Dessa maneira, possuir um forte sentimento de autoeficácia, bem como de identidade de carreira são importantes para identificar, realizar ou criar oportunidades relacionadas ao trabalho. Com isso, o trabalhador terá mais facilidade para planejar e gerenciar situações de incerteza, ou criar possibilidades que correspondam às suas aspirações (Fugate et al., 2004; Sultana \& Malik, 2020).

A idade também é uma variável com efeito significativo na percepção de empregabilidade. Pessoas mais velhas demonstram menor confiança em sua empregabilidade do que colegas mais jovens e, também, consideram-se menos empregáveis (Van der Heijden, 2002; Sok et al., 2013; Wittekind et al., 2010). Em momentos de baixa percepção de empregabilidade ou de transição de carreira, como o desemprego, a capacidade de aproveitar a identidade de carreira como um guia ao estabelecer metas e tomar decisões é crucial na identificação de oportunidades de carreira (MacArdle et al., 2007).

Adicionalmente, as conexões interpessoais são cruciais para moldar a autopercepção dos indivíduos e obter acesso a informações, oportunidades e recursos relacionados à carreira (Eby \& Lockwood, 2003). Dessa forma, a proatividade oferece aos trabalhadores uma medida de controle percebido, que aqueles com atitudes passivas ou reativas não têm (Crant, 2000). No contexto do trabalho, o capital social contribui para a empregabilidade e confere informações e influência da pessoa por meio das suas redes de contato (Adler \& Kwon, 2002).

Deve-se ressaltar a possível influência do estresse e suas implicações no bem-estar e na saúde, com a percepção de empregabilidade. A possibilidade de um indivíduo perceber uma demanda ambiental como uma ameaça ou como um desafio depende, em parte, da avaliação cognitiva dessa demanda (Lazarous \& Folkman, 1984). Trabalhadores com percepções de empregabilidade mais elevadas, por exemplo, referem como menos ameaçadoras as inseguranças ou flutuações do mercado de trabalho e, por conseguinte, sofrem menos tensão. Assim também, indivíduos que se percebem como altamente empregáveis relatam melhor saúde geral e bem-estar, e menos sintomas psicológicos (Berntson \& Marklund, 2007). Na mesma direção, a averiguação da interferência de graus de exaustão e sintomas psicológicos na percepção de empregabilidade analisada no estudo de Kinunnen et al. (2011), constatou que a percepção de empregabilidade teve implicações positivas no comportamento e no bem-estar de um indivíduo, ou seja, a percepção de empregabilidade foi associada a níveis mais baixos de exaustão no trabalho e sintomas psicológicos, e com alto desempenho e engajamento no trabalho.

A percepção de empregabilidade deve ser considerada em conjunto com os fatores contextuais (no sentido de oferta ou restrição de oportunidades de educação e trabalho), assim como o suporte de políticas públicas ou ações de responsabilidade social das 
organizações. Nesse sentido, a percepção de empregabilidade depende também do contexto de trabalho e de ações sociais que apoiem os trabalhadores na inserção ou reinserção no mercado de trabalho. O nível de empregabilidade percebida varia de acordo com o momento histórico vivido, o local onde se vive, sendo o resultado das mudanças das características pessoais e de exigências dos trabalhos disponíveis (Forrier \& Sels, 2003). Assim como a percepção de empregabilidade, o desenvolvimento de carreira exige a agência do indivíduo e é influenciado por diversos fatores contextuais. Além disso, é relevante também reconhecer o papel das crenças e das percepções do trabalhador no seu processo de tomada de decisões de carreira, em comparação com avaliações racionais e objetivas da realidade (Peixoto et al., 2015).

\section{CONSIDERAÇÕES FINAIS}

Ao longo do tempo o contexto do trabalho e das organizações percorreram inúmeras atualizações e estão constantemente submetidos a mudanças. A percepção de empregabilidade elevada se faz importante na medida em que é utilizada como um fator positivo e essencial para o progresso do trabalhador. Percepções mais fortes de empregabilidade resultam em uma perspectiva mais positiva no trabalho, e levam os trabalhadores a avaliar a insegurança ou a flexibilidade no mercado de trabalho como menos ameaçadoras. A percepção de empregabilidade está também positivamente associada não somente com à satisfação de vida, controle, otimismo, suporte no desenvolvimento e sucesso na carreira, bem como, com o engajamento, comprometimento, desempenho, satisfação e níveis mais baixos de exaustão no trabalho.

No enfoque prático, diante dos dados expostos nesse artigo, é possível fazer uma breve reflexão sobre a importância do papel dos profissionais que atuam na área de Gestão de Pessoas como ponte de apoio ao trabalhador nos seus desafios ocupacionais, bem como quais atividades esses profissionais seriam capazes de realizar em termos de intervenções para melhorar os níveis de percepção de empregabilidade do trabalhador. Nesse sentido, é possível a realização de intervenções preventivas para que o trabalhador possa usufruir de um melhor desenvolvimento em sua carreira.

A título de exemplo, pode-se usar estratégias de treinamento e desenvolvimento para desenvolver atitudes como flexibilidade, incentivar a expansão de interesses profissionais para realizar novas atividades e conquistar novos aprendizados. Sugere-se também auxiliar os trabalhadores na promoção de um senso de eficácia de enfrentamento, para reduzir o impacto das adversidades, promovendo um planejamento flexível de carreira e desenvolvendo estratégias coletivas de enfrentamento dos desafios do mercado de trabalho. Adicionalmente, a área de gestão de pessoas deve colaborar para que a organização forneça o suporte necessário aos trabalhadores, incentivando o fortalecimento das redes de segurança social, o capital social e as conexões interpessoais dos trabalhadores.

Destaca-se como limitação desse estudo a não abrangência de pesquisas científicas desenvolvidas que não tenham sido publicadas por meio de artigos em bases científicas. Este artigo teve a intenção de contribuir na disseminação do conhecimento a respeito da percepção de empregabilidade em trabalhadores. A construção de novos conhecimentos e a divulgação dessas informações, pertinentes tanto para o campo de Gestão de Pessoas, quanto para outros campos que se beneficiem dessa temática devem contribuir para compreender o sentido, significado e percepções que os trabalhadores atribuem a empregabilidade. 
Pesquisas futuras poderão averiguar, por exemplo, se os níveis baixos de percepção de empregabilidade podem explicar por que mesmo trabalhadores mais qualificados e prontos para o trabalho não conseguem emprego ou alguma atividade de trabalho e examinar o impacto das percepções de empregabilidade de trabalhadores com a demanda relativa de cada área ou de condições extremas do mercado de trabalho.

\section{REFERÊNCIAS}

Adler, P. S., \& Kwon, S. W. (2002). Social capital: Prospects for a new concept. Academy of Management Review, 27(1), 17-40. https://doi.org/10.5465/amr.2002.5922314.

Akkermans, J., Seibert, S. E., \& Mol, S. T. (2018). Tales of the unexpected: Integrating career shocks in the contemporary careers literature. SA Journal of Industrial Psychology, 44(1), 1-10. http://dx.doi.org/10.4102/sajip.v44i0.1503.

Antunes, R. (2015). The new morphology of the working class in contemporary Brazil. Socialist Register, 51(51), 178-198. https://socialistregister.com/index.php/srv/article/view/22100.

Bandura, A. (1997). Self-efficacy: The exercise of control. Macmillan.

Baruch, Y. (2001). Employability: a substitute for loyalty? Human Resource Development International, 4(4), 543-566. https://doi.org/10.1080/13678860010024518.

Berntson, E., \& Marklund, S. (2007). The relationship between perceived employability and subsequent health. Work \& Stress, 21(3), 279-292. https://doi.org/10.1080/02678370701659215.

Berntson, E., Sverke, M., \& Marklund, S. (2006). Predicting perceived employability: Human capital or labour market opportunities? Economic and Industrial Democracy, 27(2), 223- 244. https://doi.org/10.1177/0143831X06063098.

Carver, C. S., \& Scheier, M. F. (1994). Situational coping and coping dispositions in a stressful transaction. Journal of Personality and Social Psychology, 66(1), 184. https://psycnet.apa.org/fulltext/1994-29622-001.html.

Clarke, M. (2009). Plodders, pragmatists, visionaries and opportunists: Career patterns and employability. Career Development International, 14, 8-28. https://doi.org/10.1108/13620430910933556.

Crant, J. M. (2000). Proactive behavior in organizations. Journal of Management, 26(3), 435462. https://doi.org/10.1177/014920630002600304.

Cruz, R. M., Torrico, G., Knapik, J., Sales, S. S., Gai, M. J. P., Labiak, F. P., ... \& Klokner, S. G. M. (2020). Impactos da COVID-19 no trabalho e saúde mental dos trabalhadores da saúde. Research, Society and Development, 9(9), e639997783-e639997783. http://dx.doi.org/10.33448/rsd-v9i9.7783. 
De Cuyper, N., De Witte, H., Kinnunen, U., \& Natti, N. (2010). Job insecurity and employability in Finnish temporary versus permanent employees: Associations with well-being. International Studies of Management \& Organization, 40, 57-73. https://doi.org/10.1177/0894845309357051.

De Vos, A., \& Soens, N. (2008). Protean attitude and career success: The mediating role of self-management. Journal of Vocational behavior, 73(3), 449-456. https://doi.org/10.1016/j.jvb.2008.08.007.

Di Fabio, A. (2017). A review of empirical studies on employability and measures of employability. In: Psychology of Career Adaptability, Employability and Resilience. Springer, Cham, 107-123. https://doi.org/10.1007/978-3-319-66954-0_7.

Drange, I., Bernstrøm, V. H., \& Mamelund, S. E. (2018). Are you moving up or falling short? An inquiry of skills-based variation in self-perceived employability among Norwegian employees. Work, Employment and Society, 32(2), 387-406. https://doi.org/10.1177/0894845309357051.

Dries, N., Forrier, A., De Vos, A., \& Pepermans, R. (2014). Self-perceived employability, organization-rated potential, and the psychological contract. Journal of Managerial Psychology, 29(5), 565-581. https://doi.org/10.1108/JMP-04-2013-0109.

Eby, L. T., Butts, M., \& Lockwood, A. (2003). Predictors of success in the era of the boundaryless career. Journal of Organizational Behavior: The International Journal of Industrial, Occupational and Organizational Psychology and Behavior, 24(6), 689-708.

Ellinger, A. E., \& Ellinger, A. D. (2014). Leveraging human resource development expertise to improve supply chain managers' skills and competencies. European Journal of Training and Development, 38(1-2), 118-135. https://doi.org/10.1108/EJTD-09-2013-0093.

Façanha Neto, I. F., \& Façanha, J. C. R. F. (2020). Trajetória das políticas públicas de trabalho e qualificação profissional no Brasil e no Maranhão: um estudo entre os anos de 2008 a 2017. Brazilian Journal of Development, 6(3), 12304-12318.

Forrier, A., \& Sels, L. (2003). The concept employability: a complex mosaic. International Journal of Human Resources Development and Management, 3, 102-124. https://ideas.repec.org/a/ids/ijhrdm/v3y2003i2p102-124.html.

Forrier, A., Sels, L., \& Stynen, D. (2009). Career mobility at the intersection between agent and structure: A conceptual model. Journal of Occupational and Organizational Psychology, 82(4), 739-759. https://doi.org/10.1348/096317909X470933.

Fugate, M., Kinicki, A. J., \& Ashforth, B. E. (2004). Employability: A psycho-social construct, its dimensions, and applications. Journal of Vocational Behavior, 65(1), 14-38. https://doi.org/10.1016/j.jvb.2003.10.005. 
Percepção de empregabilidade de trabalhadores em condições incertas do mercado de trabalho

Gazier, B. (2006, March). Promoting employability in the context of globalisation in the EU and Japan. In 11th EU-Japan Symposium, European Commission, Japanese Ministry of Health, Labour and Welfare, Japan Institute for Labour Policy and Training, Brussels, 20-21.

Hartley, J., Jacobson, D., Klandermans, B., \& Van Vuuren, T. (1990). Job insecurity: Coping with jobs at risk. Sage Publications Ltd.

Hillage, J., \& Pollard, E. (1998). Employability: Developing a framework for policy analysis (Research Report RR85). Brighton, England: Institute for Employment Studies, Department for Education and Employment.

Hopia, H., Latvala, E., \& Liimatainen, L. (2016). Reviewing the methodology of an integrative review. Scandinavian Journal of Caring Sciences, 30(4), 662-669. https://doi.org/10.1111/scs.12327.

Jackson, D., \& Tomlinson, M. (2020). Investigating the relationship between career planning, proactivity and employability perceptions among higher education students in uncertain labour market conditions. Higher Education, 1-21.

Jackson, D., \& Wilton, N. (2017). Perceived employability among undergraduates and the importance of career self-management, work experience and individual characteristics. Higher Education Research \& Development, 36(4), 747-762. https://doi.org/10.1080/07294360.2016.1229270.

Kim, B., Jung, S. H., Jang, S. H., Lee, B., Rhee, E., Cho, S. H., \& Lee, S. M. (2014) Construction and initial validation of the Planned Happenstance Career Inventory. The Career Development Quarterly, 62(3), 239-253. https://doi.org/10.1002/j.21610045.2014.00082.x.

Kinnunen, U., Mäkikangas, A., Mauno, S., Siponen, K., \& Nätti, J. (2011). Perceived employability: Investigating outcomes among involuntary and voluntary temporary employees compared to permanent employees. Career Development International, 16(2), 140-160. https://doi.org/10.1108/13620431111115604.

Lazarus, R. S., \& Folkman, S. (1984). Stress, appraisal, and coping. Springer Publishing Company.

Lent, R. W. (2018). Future of work in the digital world: Preparing for instability and opportunity. The Career Development Quarterly, 66(3), 205-219. https://doi.org/10.1002/cdq.12143.

Makikangas, A., De Cuyper, N., Mauno, S., \& Kinnunen, U. (2013). A longitudinal personcentred view on perceived employability: The role of job insecurity. European Journal of Work and Organizational Psychology, 22(4), 490-503. 
Maranhão, N. S. M., \& Savino, T. A. C. (2019). O futuro do trabalho sob o olhar da OIT: análise do relatório "Trabalhar para um futuro melhor" (março/2019).

McArdle, S., Waters, L., Briscoe, J. P., \& Hall, D. T. T. (2007). Employability during unemployment: Adaptability, career identity and human and social capital. Journal of Vocational Behavior, 71(2), 247-264. https://doi.org/10.1016/j.jvb.2007.06.003.

Mourão, L., \& Fernandes, H. (2020). Percepção de trabalhadores acerca de inibidores e propulsores do desenvolvimento profissional. Revista Psicologia - Teoria e Prática, 22(2).

Moyo, S., \& Theron, C. (2011). A preliminary factor analytic investigation into the first-order structure of the Fifteen Plus (15FQ+) on a sample of Black South African managers. SA Journal of Industrial Psychology, 37(1), 934-956.

http://www.scielo.org.za/scielo.php?script=sci_arttext\&pid=\$207107632011000100013.

Peixoto, A. D. L. A., Janissek, J., \& Aguiar, C. V. N. (2015). Autopercepção de empregabilidade. In K. Puente-Palacios \& ADLA Peixoto. Ferramentas de diagnóstico para organizações e trabalho: um olhar a partir da psicologia, 175-186.

Qenani, E., MacDougall, N., \& Sexton, C. (2014). An empirical study of self-perceived employability: improving the prospects for student employment success in an uncertain environment. Active Learning in Higher Education, 15 (3), 199-213. https://doi.org/10.1177/1469787414544875.

Rothwell, A. T. (2015). Employability. In P. J. Hartung, M. L. Savickas, \& W. B. Walsh (Eds.), APA handbooks in psychology. APA handbook of career intervention, v. 2. Applications, 337-350. Washington, DC, US: American Psychological Association.

Rothwell, A., \& Arnold, J. (2007). Self-perceived employability: development and validation of a scale. Personnel Review, 36(1), 23-41.

https://doi.org/10.1108/00483480710716704.

Rothwell, A., Jewell, S., \& Hardie, M. (2009). Self-perceived employability: Investigating the responses of post-graduate students. Journal of Vocational Behavior, 75(2), 152-161. https://doi.org/10.1016/j.jvb.2009.05.002.

Rueda, F. J. M., Martins, L. J., \& Campos, K. C. L. (2004). Empregabilidade: o que os alunos universitários entendem sobre isto? Revista Psicologia-Teoria e Prática, 6(2). http://pepsic.bvsalud.org/scielo.php?script=sci_arttext\&pid=S151636872004000200006.

Savickas, M. L., \& Baker, D. B. (2005). The history of vocational psychology: Antecedents, origin, and early development. Handbook of Vocational Psychology, 3, 15-50. 
Percepção de empregabilidade de trabalhadores em condições incertas do mercado de trabalho

Savickas, M. L., Nota, L., Rossier, J., Dauwalder, J. P., Duarte, M. E., Guichard, J., \& Van Vianen, A. E. (2009). Life designing: A paradigm for career construction in the 21st century. Journal of Vocational Behavior, 75(3), 239-250. https://doi.org/10.1111/j.1556-6676.2012.00002.x.

Sok, J., Blomme, R., \& Tromp, D. (2013). The use of the psychological contract to explain selfperceived employability. International Journal of Hospitality Management, 34, 274284. https://doi.org/10.1016/j.ijhm.2013.03.008.

Suleman, F. (2018). The employability skills of higher education graduates: insights into conceptual frameworks and methodological options. Higher Education, 76(2), 263-278. https://doi.org/10.1007/s10734-017-0207-0.

Sultana, R., \& Malik, O. F. (2020). Protean career attitude, perceived internal employability and perceived external employability; does self-efficacy make a difference? Middle East Journal of Management, 7(4), 343-364. https://doi.org/10.1504/MEJM.2020.108076.

Thijssen, J. G., Van der Heijden, B. I., \& Rocco, T. S. (2008). Toward the employability - link model: current employment transition to future employment perspectives. Human Resource Development Review, 7(2), 165-183. https://doi.org/10.1177/1534484308314955.

Torraco, R. J. (2016). Writing integrative literature reviews: Using the past and present to explore the future. Human Resource Development Review, 15(4), 404-428. https://doi.org/10.1177/1534484316671606.

Tsitskari, E., Goudas, M., Tsalouchou, E., \& Michalopoulou, M. (2017). Employers' expectations of the employability skills needed in the sport and recreation environment. Journal of Hospitality, Leisure, Sport \& Tourism Education, 20, 1-9. https://doi.org/10.1016/j.jhlste.2016.11.002.

Van Dam, K. (2004). Antecedents and consequences of employability orientation. European Journal of Work and Organizational Psychology, 13, 29-51. https://doi.org/10.1080/13594320344000237.

Van der Heijde, C. M. (2014). Employability and self-regulation in contemporary careers. In: Psycho-social career meta-capacities, 7-18. Springer, Cham. https://doi.org/10.1007/978-3-319-00645-1_1.

Van der Heijden, B. (2002). Prerequisites to guarantee life-long employability. Personnel Review. https://doi.org/10.1108/00483480210412418.

Van der Klink, J. J., Bültmann, U., Burdorf, A., Schaufeli, W. B., Zijlstra, F. R., Abma, F. I., ... \& Van der Wilt, G. J. (2016). Sustainable employability-Definition, conceptualization, and implications: A perspective based on the capability approach. Scandinavian Journal of Work, Environment \& Health, 71-79. https://www.jstor.org/stable/43999197. 
Veld, M., Semeijn, J., \& van Vuuren, T. (2015). Enhancing perceived employability. Personnel Review, 44(6), 866. https://doi.org/10.1108/PR-05-2014-0100.

Veth, K. N., Emans, B. J., Van der Heijden, B. I., Korzilius, H. P., \& De Lange, A. H. (2015). Development (f) or maintenance? An empirical study on the use of and need for HR practices to retain older workers in health care organizations. Human Resource Development Quarterly, 26(1), 53-80. https://doi.org/10.1002/hrdq.21200.

Wittekind, A., Raeder, S., \& Grote, G. (2010). A longitudinal study of determinants of perceived employability. Journal of Organizational Behavior, 31(4), 566-586. 